\title{
Identification of sympatric cryptic species of Aedes albopictus subgroup in Vietnam: new perspectives in phylosymbiosis of insect vector
}

\author{
Guillaume Minard ${ }^{1,2,3,4,5^{*}}$, Van Tran Van ${ }^{1,2,3,4}$, Florence Hélène Tran ${ }^{1,2,3,4^{\wedge}}$, Christian Melaun ${ }^{6,7}$, Sven Klimpel ${ }^{6,7}$, \\ Lisa Katharina Koch ${ }^{6,7}$, Khanh Ly Huynh Kim ${ }^{8}$, Trang Huynh Thi Thuy ${ }^{8}$, Huu Tran Ngoc ${ }^{8}$, Patrick Potier ${ }^{1,2,3,4}$, \\ Patrick Mavingui ${ }^{1,2,3,4,9}$ and Claire Valiente Moro ${ }^{1,2,3,4}$
}

\begin{abstract}
Background: The Aedes (Stegomyia) albopictus subgroup includes 11 cryptic species of which Ae. albopictus is the most widely distributed. Its global expansion associated with a documented vector competence for several emerging arboviruses raise obvious concerns in the recently colonized regions. While several studies have provided important insights regarding medical importance of Ae. albopicus, the investigations of the other sibling species are scarce. In Asia, indigenous populations within the Ae. albopictus subgroup can be found in sympatry. In the present study, we aimed to describe and compare molecular, morphological and bacterial symbionts composition among sympatric individuals from the Ae. albopictus subgroup inhabiting a Vietnamese protected area.

Results: Based on morphological structure of the cibarial armarture, we identified a cryptic species in the forest park at Bù Gia Mập in the south-eastern region of Vietnam. Analysis of nuclear (ITS1-5.8S-ITS2) and mitochondrial (cox1, nad5) markers confirmed the divergence between the cryptic species and Ae. albopictus. Analysis of midgut bacterial microbiota revealed a strong similarity among the two species with a notable difference; contrary to Ae. albopictus, the cryptic species did not harbour any Wolbachia infection.

Conclusions: These results could reflect either a recent invasion of Wolbachia in Ae. albopictus or alternatively a loss of this symbiont in the cryptic species. We argue that neglected species of the Ae. albopictus subgroup are of main importance in order to estimate variation of host-symbionts interactions across evolution.
\end{abstract}

Keywords: Asian tiger mosquito, Sibling species, Wolbachia, Microbiota, Dysgonomonas

\section{Background}

The Aedes subgenus Stegomyia contains currently 128 species [1]. Among them, Aedes aegypti and Ae. albopictus are of main public health concern. They are considered as main vectors for dengue and chikungunya (DENV, CHIKV) as well as Zika fever viruses, all infectious to humans. These mosquito species have also been evidenced

\footnotetext{
* Correspondence:

guillaume.minard@helsinki.fi; guillaume_minard_86@hotmail.com

Deceased

'Université de Lyon, Lyon, France

Université Lyon 1, Villeurbanne, France

Full list of author information is available at the end of the article
}

as potential vectors under laboratory conditions for a wide range of other arboviruses including Japanese encephalitis virus, West Nile virus, eastern equine encephalitis virus and La Crosse virus. Nevertheless, the involvement of these mosquito species in the transmission of these viruses remains to be demonstrated in the field.

Identification of species within the subgenus Stegomyia is often based on morphological features and in particular, for adults, on patterns on the thorax (especially the scutum) and tarsi [2-5]. However, these morphological characters are not sufficient to distinguish some species, which may lead to misidentification of individuals collected in the field. This is significant for Ae. albopictus 
sibling species, combined in the literature as members of the Scutellaris group and Ae. albopictus subgroup. These species have very similar morphological characteristics especially at the larval and adult (females) stages $[2-4,6]$. Furthermore, although some of the species of this subgroup have different ecological niches, some of them are found in sympatry [3, 7-9]. In Asia, indigenous populations of Ae. albopictus coexist with populations of Ae. flavopictus, Ae. pseudalbopictus, Ae. subalbopictus, Ae. patricae, Ae. seatoi and Ae. novalbopictus [3, 7]. In Greece, invasive populations of Ae. albopictus occur in sympatry with indigenous populations of Ae. cretinus [9]. The importance of the species of the subgroup Ae. albopictus in disease transmission has been poorly studied so far. This can be explained by low contact levels of these species with human populations as well as by their high resemblance to the Asian tiger mosquito, potentially leading to misidentification.

Sympatric cryptic species with recent divergences constitutes a privilege system for the understanding of symbiosis evolution. The study of symbiotic interactions is a complex and dynamic system and previous experiments have revealed strong variations in symbionts composition when comparing laboratory-reared $v s$ fieldcaught mosquitoes but also among individuals caught in different ecosystems [10-13]. These modifications can be explained by host or symbiont population dynamics (genetic drift, bottleneck effect, expansion), modification of symbionts transmission-acquisition probability but also by modification of nutrients quality or abiotic factors that could suggest a local adaptation of one or both partners and local variation of their interaction [14-16]. The bacterial microbiota of Ae. albopictus presents a relative homogenous structure among populations and studies on whole body from field-caught individuals highlighted a dominance of Wolbachia pipientis wAlbA and $w \mathrm{AlbB}$ which often co-occur at a prevalence of $\sim 100 \%$ [17-22]. These intracellular symbionts are involved in a control of the reproduction of Ae. albopictus through cytoplasmic incompatibility [23]. This process results from aberrant offspring production between infected males and uninfected females, or between hosts carrying incompatible Wolbachia strains. In insects, this control of reproductive process has been proposed to be the cause of reproductive isolation between populations [24]. Moreover, long term infections with Wolbachia and prevalence variation among populations could participate in speciation events. Similarly, reproductive isolation can be a barrier to the invasion of Wolbachia [25]. Studies conducted among parasitoid wasps Nasonia giraulti and $N$. vitripennis also showed that bacterial microbiota could be involved in speciation resulting from reproductive isolations [26]. Such events are more susceptible to occur in species complexes that have recently diverged and therefore lead to asymmetric symbionts composition regardless of their relative genetic similarity. Microbial community divergences occurring in the midgut of mosquitoes could directly impact the ecophysiology of this organ, and to a large extent the vectorial capacities of mosquitoes. Indeed, the replication of virus pathogens through the midgut constitutes the first bottleneck affecting the diversity and density of the particles [27, 28]. Furthermore, recent advances demonstrated that bacterial symbionts participate to these bottlenecks by: (i) immune priming, (ii) resource competition, and (iii) secondary metabolites production [29].

In the Nature Reserve of Bù Gia Mập in south-eastern Vietnam, we found a cryptic species living in sympatry with Ae. albopictus. Description of cryptic species that have recently diverged is important to decipher the recent evolution of traits in medically important vectors. We were especially interested in describing the symbiotic associations among sympatric cryptic species as variations in those associations might help to disentangle the history of symbiotic invasions as well as loss of interactions between host and symbionts. To assess its proximity to the tiger mosquito, we genotyped individuals using mitochondrial and nuclear markers and analysed their associated bacterial microbiota.

\section{Results \\ Morphological and molecular features reveal differences among Ae. albopictus living in sympatry}

Adult female mosquitoes collected in the field were all identified as members of the Ae. albopictus subgroup using various morphological characters, especially the line of pale scale in the posterior scutum, a broad patch of white scales across lateral face of scutellum (Fig. 1). Since such morphological identification is notoriously insufficient to separate adult females within the Ae. albopictus subgroup [2, 3, 7], we completed the identification using molecular markers. The phylogenetic distances between individuals were estimated through two mitochondrial (cox1, nad5) and three nuclear (ITS1-5.8S-ITS2 rRNA) barcodes over 43 individuals (Table 1). For technical reason 48 individuals have been haplotyped with the $\operatorname{cox} 1$ marker, 44 of those were also haplotyped with the ND5 marker, and 25 of them were genotyped with ITS1-5.8S-ITS2 marker. Individuals displaying genetically close barcodes were clustered under a unique reference haplotype number (Table 2), leading to a total of 21,7 and 25 different reference haplotypes using cox 1 , nad5 and ITS1-5.8SITS2 markers, respectively. Distances among reference haplotypes estimated with Kimura 2-parameter (K2P) were highly correlated between the different barcodes (cox1 - ITS1-5.8S-ITS2, $r_{(274)}=0.99, P<2.2 .10^{-16} ; \operatorname{cox} 1$ - 

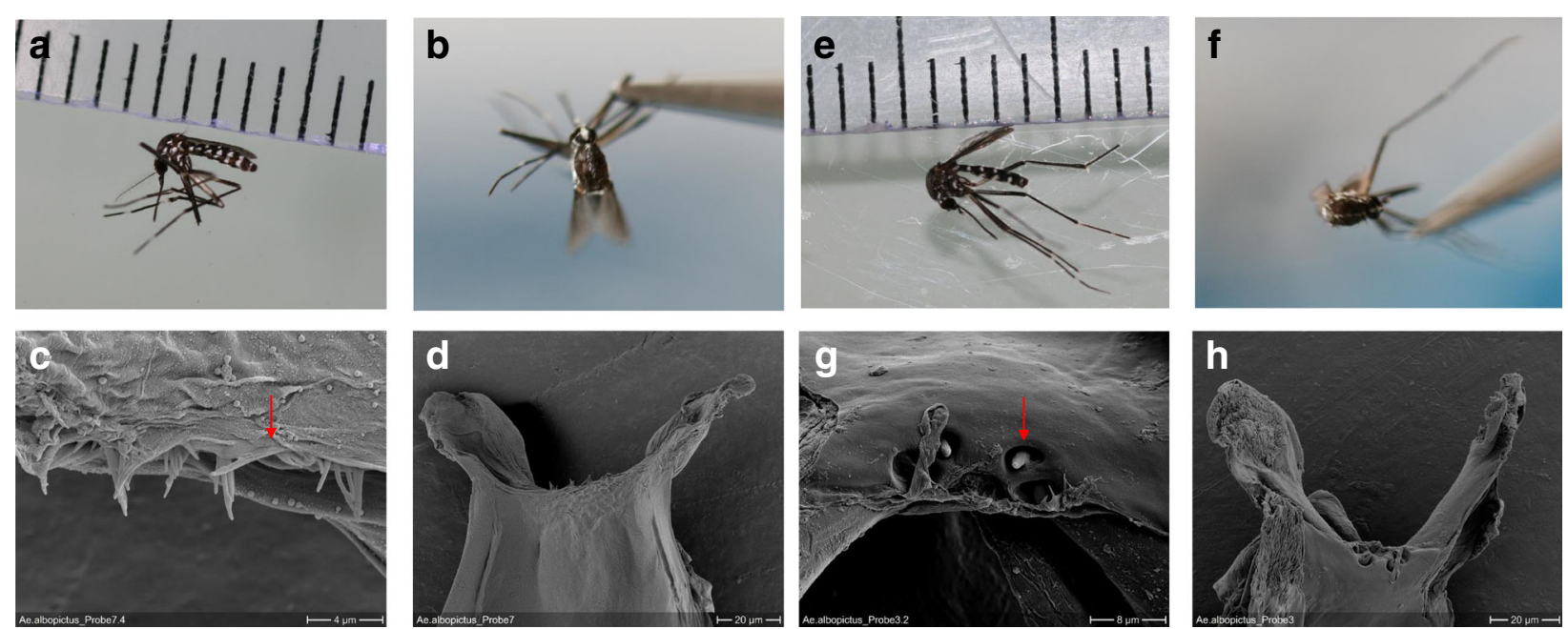

Fig. 1 Composite figure of morphological comparison of the cryptic species Aedes sp. and Ae. albopictus from Bù Gia Mập National Park. Cryptic species Aedes sp:: a Lateral view. b Scutum. c Scanning electron microscopy of the ventral papillae. Cibarial teeth are absent (arrow). d Scanning electron microscopy of the cibarial armature including the lateral flange. Aedes albopictus: e Lateral view. f Scutum. g Scanning electron microscopy of the ventral papillae and four short cibarial teeth (arrow). $\mathbf{h}$ Scanning electron microscopy of the cibarial armature including the lateral fringe

nad5, $r_{(701)}=0.99, P<2.2 .10^{-16} ;$ nad5 - ITS1-5.8S-ITS2, $r_{(204)}=0.99, P<2.2 .10^{-16}$ ) (Additional file 1: Figure S1). Two congruent monophyletic groups were identified subsequently and assigned to two different species (Table 2, Figs. 2, 3 and 4). The two clades were called 'A' and ' $\mathrm{C}$ ' and corresponded respectively to the species Ae. albopictus and to a non-characterized cryptic species hereafter referred as Aedes sp. Both taxa could be discriminated using polymorphism at ITS1-5.8S-ITS2 locus. The individuals associated to clade A had an amplicon size $>1150$ bp whereas those associated with clade $C$ had an approximate amplicon size $<1050 \mathrm{bp}$, allowing easy and accurate identification of both clades through $1 \%$ agarose gel electrophoresis (Additional file 1: Figure S2). It is however important to mention that this PCR could lead to an additional band of $<560$ bp in Ae. albopictus depending on the presence of the symbiotic protist Ascogregarina taiwannensis, which did not interfere with the ITS-based identification of A and C. Among those three markers, individuals from the clade $\mathrm{C}$ presented an equivalent or slightly higher haplotype diversity (Hdcox $1=0.74$; Hdnad $5=0.23$; HdITS1-5.8S-ITS2 = 1) than the individuals from the clade A (Hdcox $1=0.70 ; \operatorname{Hd} \operatorname{nad} 5=0.20$; HdITS1-5.8S-ITS2 =1). Two $\operatorname{cox} 1$ sequences annotated as

Table 1 Samples information

\begin{tabular}{ll}
\hline & $\begin{array}{l}\text { Number of tested individuals } \\
\text { (Ae. albopictus/cryptic Aedes sp.) }\end{array}$ \\
\hline Barcoding and Wolbachia detection & $43(17 / 26)$ \\
b-ARISA & $24(9 / 15)$ \\
16SrDNA MiSeq sequencing & $5(3 / 2)$ \\
Total & $72(29 / 43)$ \\
\hline
\end{tabular}

Ae. albopictus but close the the clade $\mathrm{C}$ were found in the public databases (Additional file 1: Supplementary informations). In order to improve detection methods for Aedes sp., observation of cibarial armarture through scanning electron microscopy was used to highlight any morphological difference between members of clades A and $C$. Three morphological differences were characterized for both species: (i) a structural difference in the ventral papillae; (ii) a difference in the angle of the lateral flange; and (iii) the cibarial teeth. While all ventral papillae in Ae. albopictus become continuously slimmer from the base to tip, the ones in Aedes sp. from Bù Gia Mập show a bulbous thickening before the tip; the angle of the lateral flanges is wider in the cibarium of Aedes sp. from Bù Gia Mập than in the one of Ae. albopictus; cibarial teeth are absent in Aedes sp. from Bù Gia M p, while Ae. albopictus has four short cibarial teeth (Fig. 1). Therefore, those morphological keys can be used to differentiate the two species.

\section{Wolbachia are naturally present in Ae. albopictus but absent in the cryptic species}

Among all individuals from clade A, assigned to Ae. albopictus, all tested individuals (17/17) were diagnosed positive for Wolbachia infection via amplification of the wsp gene (Additional file 1: Table S1). On the contrary, no Wolbachia $(0 / 26)$ were detected in the individuals from clade C (cryptic Aedes sp.).

\section{Midgut bacterial microbiota are similar between $A e$. albopictus and the cryptic species} b-ARISA analysis of mosquito midgut microbiota was performed on a total of 24 individuals. Using ITS1-5.8S- 
Table 2 Individual's haplotypes and Wolbachia infection status

\begin{tabular}{|c|c|c|c|c|}
\hline Sample reference & cox1 clade (Haplotype) & nad5 & ITS1-5.8S-ITS2 & Wolbachia - wsp \\
\hline 1 & Clade A (Hap_2) & Clade A (Hap_1) & - & positive \\
\hline 2 & Clade A (Hap_2) & Clade A (Hap_1) & - & positive \\
\hline 3 & Clade A (Hap_2) & Clade A (Hap_1) & Clade A (Hap_1) & positive \\
\hline 4 & Clade C (Hap_1) & Clade C (Hap_3) & Clade C (Hap_12) & negative \\
\hline 5 & Clade C (Hap_5) & Clade C (Hap_3) & Clade C (Hap_14) & negative \\
\hline 6 & Clade A (Hap_2) & - & Clade A (Hap_4) & positive \\
\hline 7 & Clade C (Hap_9) & Clade C (Hap_3) & Clade C (Hap_17) & negative \\
\hline 8 & Clade C (Hap_5) & Clade C (Hap_3) & Clade C (Hap_21) & negative \\
\hline 9 & Clade A (Hap_7) & Clade A (Hap_1) & Clade A (Hap_5) & positive \\
\hline 10 & Clade A (Hap_8) & Clade A (Hap_2) & - & positive \\
\hline 11 & Clade A (Hap_2) & Clade A (Hap_1) & - & positive \\
\hline 12 & Clade A (Hap_2) & Clade A (Hap_1) & Clade A (Hap_3) & positive \\
\hline 13 & Clade A (Hap_2) & Clade A (Hap_5) & - & positive \\
\hline 14 & Clade A (Hap_7) & Clade A (Hap_1) & - & positive \\
\hline 15 & Clade C (Hap_6) & Clade C (Hap_3) & - & negative \\
\hline 16 & Clade C (Hap_5) & Clade C (Hap_3) & Clade C (Hap_10) & negative \\
\hline 17 & Clade A (Hap_2) & Clade A (Hap_1) & - & positive \\
\hline 18 & Clade C (Hap_5) & Clade C (Hap_3) & Clade C (Hap_25) & negative \\
\hline 19 & Clade C (Hap_5) & Clade C (Hap_3) & Clade C (Hap_7) & negative \\
\hline 20 & Clade C (Hap_10) & Clade C (Hap_6) & Clade C (Hap_9) & negative \\
\hline 21 & Clade A (Hap_3) & Clade A (Hap_1) & - & positive \\
\hline 22 & Clade C (Hap_4) & - & Clade C (Hap_13) & negative \\
\hline 23 & Clade A (Hap_12) & Clade A (Hap_1) & - & positive \\
\hline 24 & Clade C (Hap_5) & Clade C (Hap_3) & - & negative \\
\hline 25 & Clade C (Hap_5) & Clade C (Hap_3) & Clade C (Hap_6) & negative \\
\hline 26 & Clade C (Hap_5) & Clade C (Hap_3) & Clade C (Hap_8) & negative \\
\hline 27 & Clade C (Hap_5) & Clade C (Hap_3) & - & negative \\
\hline 28 & Clade A (Hap_18) & Clade A (Hap_1) & Clade A (Hap_2) & positive \\
\hline 29 & Clade A (Hap_19) & Clade A (Hap_1) & - & positive \\
\hline 30 & Clade C (Hap_20) & - & Clade C (Hap_19) & negative \\
\hline 31 & Clade C (Hap_5) & Clade C (Hap_3) & Clade C (Hap_23) & negative \\
\hline 32 & Clade C (Hap_21) & Clade C (Hap_7) & - & negative \\
\hline 33 & Clade C (Hap_13) & Clade C (Hap_3) & Clade C (Hap_18) & negative \\
\hline 34 & Clade C (Hap_13) & Clade C (Hap_3) & Clade C (Hap_22) & negative \\
\hline 35 & Clade C (Hap_13) & Clade C (Hap_3) & - & negative \\
\hline 36 & Clade C (Hap_14) & Clade C (Hap_3) & - & negative \\
\hline 37 & Clade C (Hap_13) & - & Clade C (Hap_11) & negative \\
\hline 38 & Clade C (Hap_5) & Clade C (Hap_3) & Clade C (Hap_26) & negative \\
\hline 39 & Clade A (Hap_2) & Clade A (Hap_1) & - & positive \\
\hline 40 & Clade C (Hap_15) & Clade C (Hap_3) & Clade C (Hap_16) & negative \\
\hline 41 & Clade C (Hap_5) & Clade C (Hap_3) & Clade C (Hap_20) & negative \\
\hline 42 & Clade C (Hap_16) & Clade C (Hap_4) & Clade C (Hap_24) & negative \\
\hline 43 & Clade A (Hap 17) & Clade A (Hap 1) & - & positive \\
\hline
\end{tabular}


Table 2 Individual's haplotypes and Wolbachia infection status (Continued)

\begin{tabular}{|c|c|c|c|c|}
\hline $\mathrm{BGM}^{\mathrm{a}}$ & Clade A (Hap_2) & Clade A (Hap_1) & - & - \\
\hline $\mathrm{BGM}^{\mathrm{a}}$ & Clade A (Hap_2) & Clade A (Hap_1) & - & - \\
\hline $\mathrm{BGM} 4^{\mathrm{a}}$ & Clade A (Hap_11) & Clade A (Hap_1) & - & - \\
\hline $\mathrm{BGM}^{\mathrm{a}}$ & Clade C (Hap_5) & Clade C (Hap_3) & - & - \\
\hline$B G M 6^{\mathrm{a}}$ & Clade C (Hap_5) & Clade C (Hap_3) & - & - \\
\hline
\end{tabular}

${ }^{\mathrm{a} S a m p l e s ~ u s e d ~ f o r ~ m i c r o b i o t a ~ a n a l y s i s ~}$

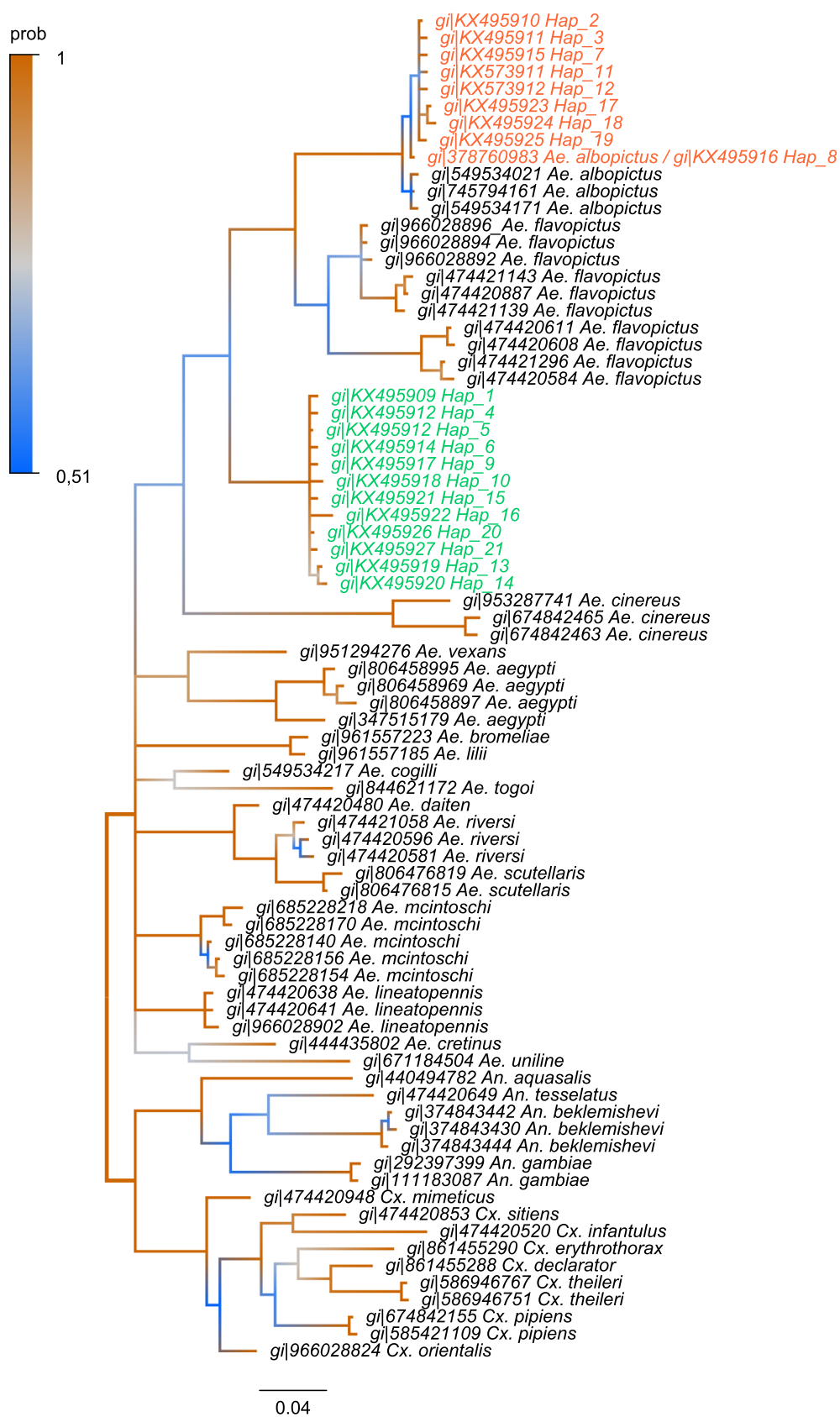

Fig. 2 Phylogenetic tree based on cox1 sequences for species of the Culicidae. Bayesian consensus tree is represented. Phylogeny was built using the GTR $+I+\Gamma$ evolution model. Branches are coloured according to their posterior probability (prob). Clade 'A' associated with Ae. albopictus (s.s.) is coloured in red. Clade ' $C$ ' associated with cryptic species of Ae. albopictus subgroup is coloured in green 


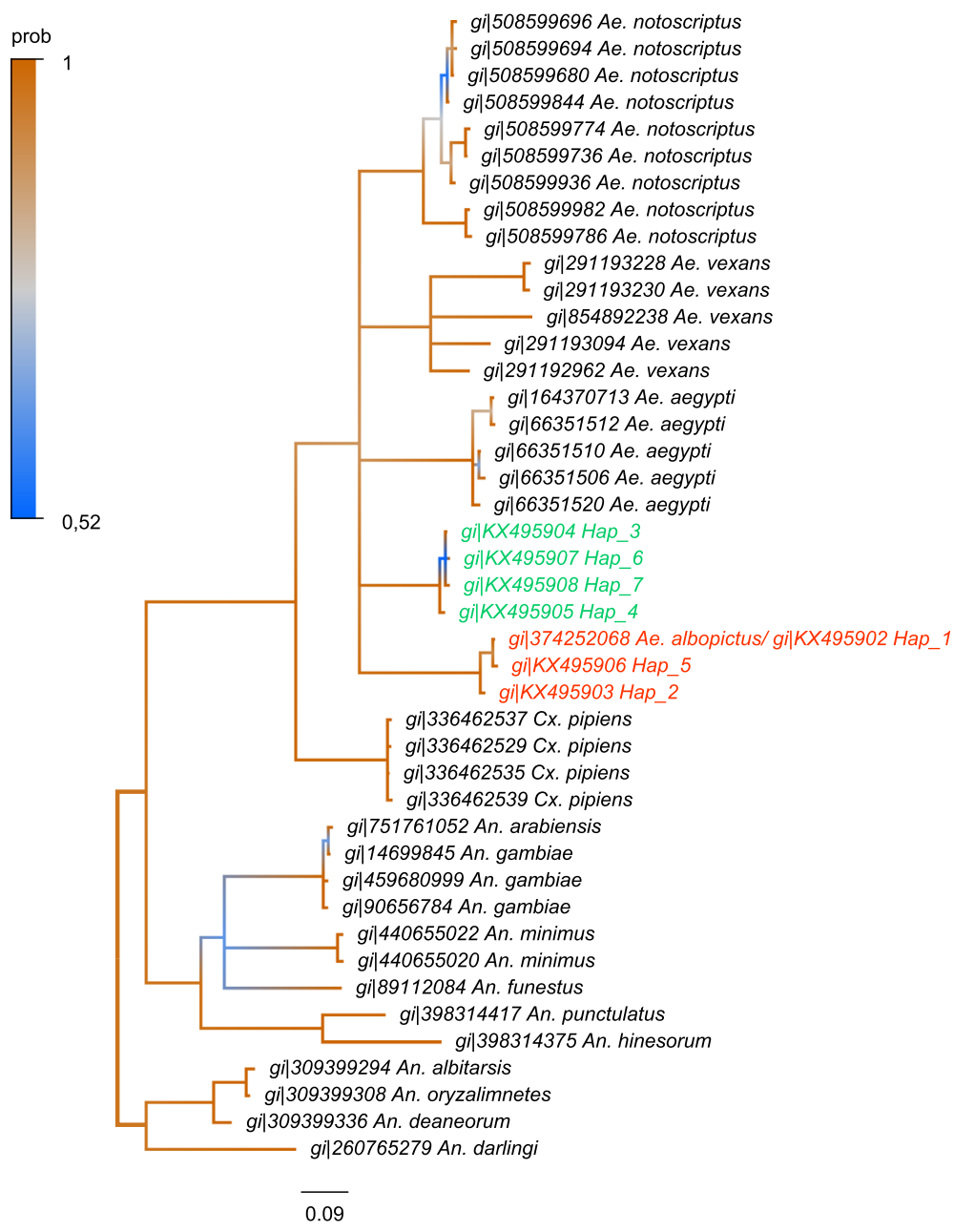

Fig. 3 Phylogenetic tree based on nad5 sequences for species of the Culicidae. Bayesian consensus tree is represented. Phylogeny was built using the HKY $+I+\lceil$ evolution model. Node labels refer to posterior probability of the separation. Branches are coloured according to their posterior probability (prob). Clade ' $\mathrm{A}$ ' associated with Ae. albopictus (s.s.) is coloured in red. Clade ' $\mathrm{C}$ ' associated with cryptic species of Ae. albopictus subgroup is coloured in green

ITS2 polymorphism presented above, a total of 9 individuals were assigned to the A clade of Ae. albopictus species and 15 individuals to the cryptic Aedes sp. species (i.e. clade C). The Shannon $\alpha$-diversity index was equivalent between both species (Mann-WhitneyWilcoxon, $U_{(34)}=102, Z=-0.04, P=0.7$ ) (Fig. 5a). Computing Bray-Curtis dissimilarity distance, no divergence in bacterial community structure was observed between the two species (adonisANOVA, $F_{(1,32)}=1.02, P=0.37$ ) (Fig. $5 \mathrm{~b}$ ). In order to assess the taxonomic composition of bacterial community between the two species, a metabarcoding analysis was performed on a set of 5 additional individuals. The seventeen dominant operational taxonomic units (OTUs), which represented more than $1 \%$ of the overall rarefied sequences were represented (Fig. 6). Interestingly, the dominant taxa were similar between cryptic specimens and Ae. albopictus specimens.
Dysgonomas sp., as well as members of the family Sphingomonadaceae (Sphingobium, Novosphingobium, Sphingomonas) represented more than $35 \%$ of the sequences among the individuals (Additional file 1: Table S2).

\section{Discussion}

To the best of our knowledge, in this study we report for the first time the presence of a phylogenetically divergent cryptic species living in sympatry with $A e$. albopictus in a protected forest of the Binh Phuoc Province in Vietnam. For convenience, no name has been given to this species. Since no mating experiments have been performed, further investigations would be required to confirm the reproductive isolation of the two species. We used several markers (barcodes, amplicon length, cibarial armature morphology) that could be used later to discriminate the cryptic species from $A e$. albopictus (sensu stricto). These markers constitute a 


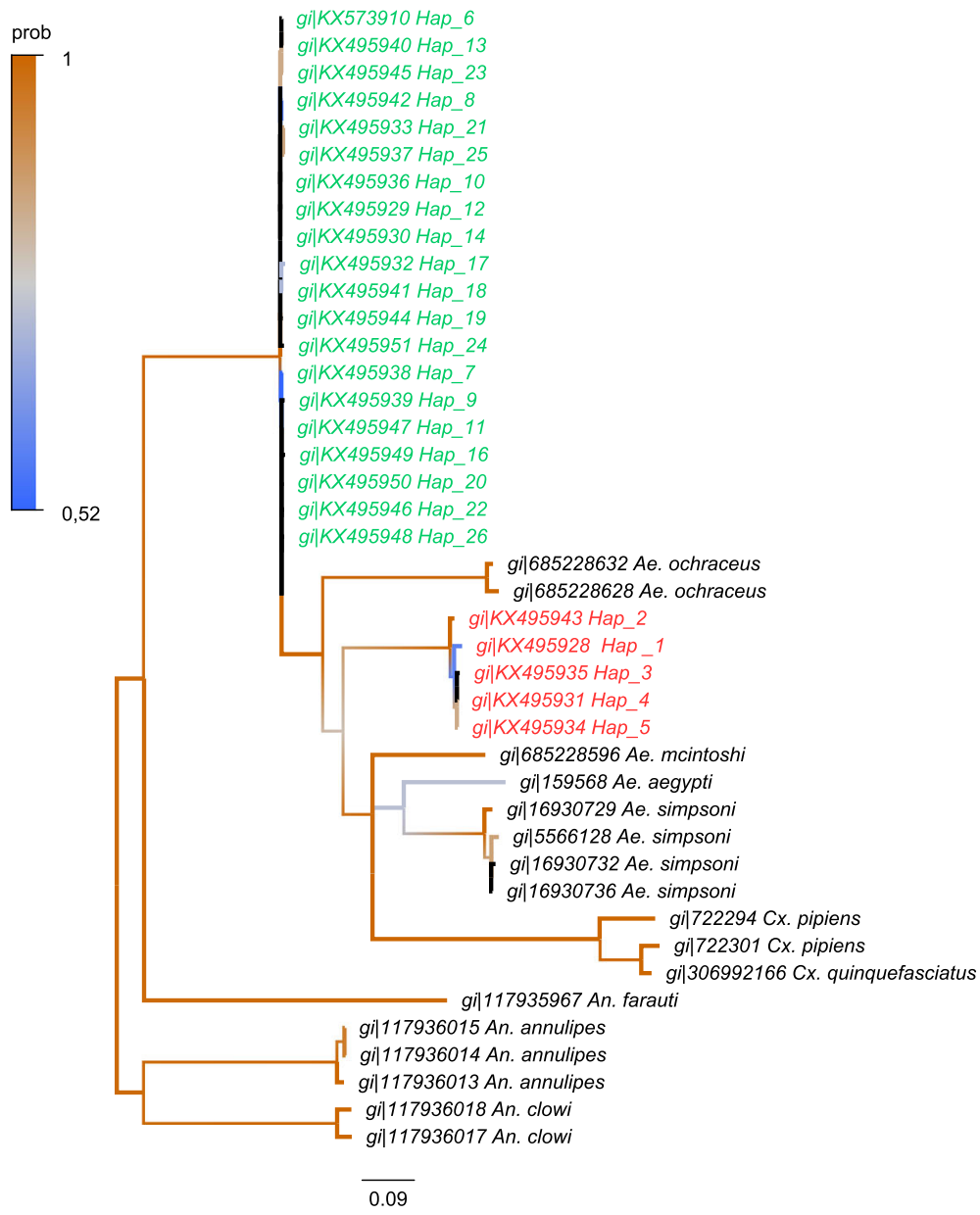

Fig. 4 Phylogenetic tree based on ITS1-5.8S-ITS2 sequences for species of the Culicidae. Bayesian consensus tree is represented. Phylogeny was built using the $\mathrm{HKY}+\mathrm{I}+\Gamma$ evolution model. Node labels refer to posterior probability of the separation. Branches are coloured according to their posterior probability (prob). Clade ' $A$ ' associated with Ae. albopictus (s.s.) is coloured in red. Clade ' $C$ ' associated with cryptic species of Ae. albopictus subgroup is coloured in green
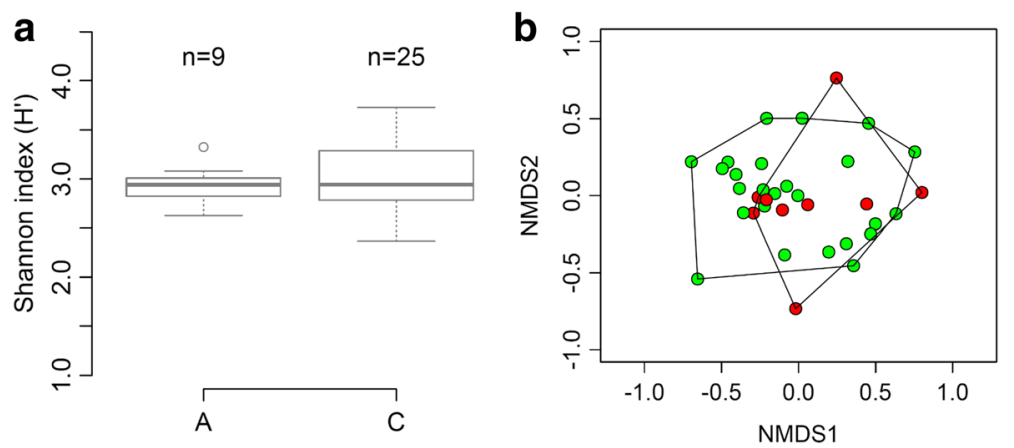

Fig. 5 Comparison of bacterial diversity between individuals of the two Ae. albopictus cryptic species. a Boxplot representation of Shannon a-diversity within individuals associated to the ' $A$ ' clade (Ae. albopictus) and the ' $C$ ' clade (cryptic species). b 2D non-metric multidimensional sequence scaling representing dissimilarity distances among individuals of the ' $A$ ' clade in green and the ' $C$ ' clade in red 


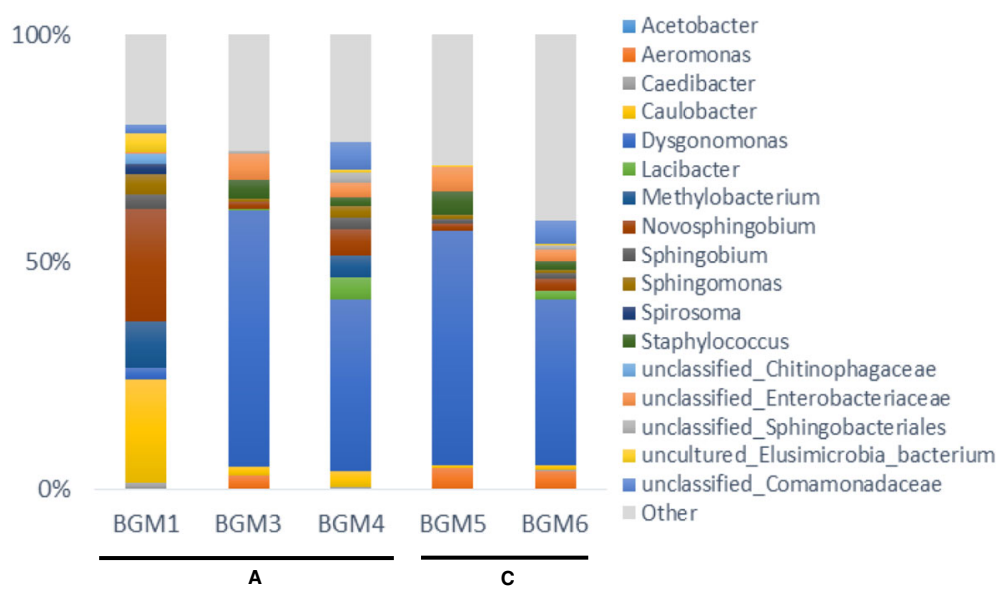

Fig. 6 Bacterial composition of the midgut of the two Ae. albopictus cryptic species. The proportion of each taxon refers to the proportion of sequences identified through V5-V6 regions of 16S rDNA sequenced with MiSeq. The taxa names refer to assignation of 3\% distance OTUs inside the samples. The taxa, which represented less than $1 \%$ of the sequences, were classified as "other". Five individuals were analysed and belonged respectively to the ' $A$ ' clade (Ae. albopictus) or the ' $C$ ' clade (cryptic species)

useful tool for entomological studies as we report previous inconsistent and presumably wrong assignation of this species among Ae. albopictus subgroup based solely on cox 1 barcodes (Additional file 1: Supplementary information). Using similar approaches, previous investigations on Anopheles malaria vectors highlighted the presence of various cryptic species in Vietnamese national parks [30]. Characterization of vector species is a prerequisite to estimate pathogens transmission risks. In South-East Asia, identification of Aedes mosquitoes remains of main concern because of their ability to replicate and retransmit viral pathogens including dengue or Japanese encephalitis viruses. The Province of Binh Phuoc in Vietnam has often been identified as an advanced region for dengue transmission suggesting that this location could be a centroid of annual epidemics [31]. Two main identified vectors are involved in replication and transmission of this virus in South-East Asia: Ae. aegypti and Ae. albopictus. Of the latter, various cryptic species occur in that region $[3,7,8]$. However, the ecology of these species is partially unknown, at least in part, because of diagnosis difficulties. In addition to their epidemiological interest, we argue that studies on the ecology of sympatric species sharing a recent last common ancestor constitute a privileged system for the understanding of evolutionary processes. Symbiosis, in particular is a complex investigation field as the nature of interactions between host and microbes can drastically change across evolution depending on changes in: (i) trophic interactions; (ii) symbionts transmission pathways among hosts; and (iii) coevolution.

In this study, we were not able to detect any Wolbachia infection in the cryptic species of the Ae. albopictus complex. The difference in infection status between these related species is interesting as Wolbachia-induced sexual isolation could occur before hybrid lethality and therefore participate to speciation events [24]. In addition to their consistent divergence, individuals from the clade $\mathrm{C}$ lacking Wolbachia infections presented a slightly higher haplotype diversity relative to these from the clade A. Several examples in the Culex pipiens complex witnessed an asymmetric invasion by Wolbachia within cryptic species [32-34]. Indeed, non-invaded populations of $C x$. pipiens from South Africa harbored an ancestral and higher mitochondrial diversity [32]. This reduction of organelle genetic diversity often occurs after Wolbachia invasion due to mitochondrial hitchhiking successive to the rapid spread of the symbiont [35]. Such mitochondrial sweep was not supported by our data as no significant differences in the haplotypes diversities were observed between Ae. albopictus and the cryptic species. A recent study, conducted by Dumas et al. [34] highlighted a similar divergence across nuclear and mitochondrial genomes when comparing uninfected and infected $C x$. pipiens populations. This nuclear divergence supported the hypothesis of a reproductive isolation among those sympatric individuals that did not present any gene flow among them [34]. Similarly, a haplotype-based study of fig wasp Eupristina verticillata conducted within two Chinese provinces demonstrated that morphologically similar individuals diverged in three cryptic species [36]. These observations were supported with both mitochondrial and nuclear markers and were correlated with the Wolbachia clades infecting them. The authors suggested that a reproductive manipulation induced by Wolbachia might have been responsible for the observed speciation. Such patterns contrast with 
Wolbachia related-genetic divergences that were observed in lepidopterans Phengaris teleius and P. nausithious [37]. Within these two species, individuals infected by Wolbachia presented a mitochondrial divergence without consistent nuclear genetic structure. Such cryptic speciation mimicry might be induced by a mitochondrial hitchhiking without any gene flow barrier preventing breeding of infected and uninfected individuals. The pattern observed in our study supported a mitochondrial and nuclear divergence among the two sympatric $\mathrm{A}$ and $\mathrm{C}$ clades, which is consistent with a cryptic speciation. Similarly to the previous examples, our results do not inform whether a Wolbachia-independent sexual isolation also occurred. Mating experiments of cryptic species with Wolbachia infected or uninfected lines could enable us to estimate whether hybrid lethality or Wolbachia-induced sexual isolation occurred between these species.

In addition, our b-ARISA analysis showed that Ae. albopictus midgut microbiota does not differ from that of the cryptic species. MiSeq sequencing of $16 \mathrm{~S}$ rDNA variants of 3 individuals of Ae. albopictus and 2 individuals of the cryptic species identified Dysgonomonas and Sphingomonadaceae as potential dominant taxa of the bacterial microbiota. The bacterial composition of Ae. albopictus midgut is strongly different from the rest of its body [22]. Investigating midgut microbiota of seven populations from France and Vietnam, we previously discovered a similar structure across populations with the presence of the same dominant genus Dysgonomonas sp. [22]. Individuals sampled in Vietnam also showed an enrichment of several taxa including Sphingomonadaceae (Sphingobium, Sphingomonas, Novosphingobium). Most of these similar bacterial taxa are also found in the water and plants with which mosquitoes are in contact during immature and adult stages [38, 39]. A recent study on the Litoditis marina complex (Nematoda) described a divergent structure between the bacterial microbiota among three cryptic species [40]. The authors suggested a link between the microbiota, intrinsic physiological properties and trophic interactions specificities for each species. Contrary to these results, the similarity between midgut bacterial microbiota associated with the two species of the Ae. albopictus complex revealed here, suggests similar physiology and trophic interactions with plants and vertebrate' hosts. However, further investigations would be needed in order to properly describe the taxonomic composition of the bacterial communities associated with the midgut of Ae. albopictus cryptic species compared to that of Ae. albopictus.

\section{Conclusion}

The identification of a cryptic species of Ae. albopictus in Vietnamese forest was evidenced by the detection of genetically divergent and morphologically similar individuals. New morphological keys based on cibarial armature and papillae were proposed for the distinction of this species from Ae. albopictus (s.s). Intestinal microbiota of both species were equivalent suggesting a similar selection - acquisition of their digestive symbionts. However, both species differed by their Wolbachia infection status. The two species present a promising study system to investigate the relationship between symbionts and vector insects evolving in equivalent ecosystems.

\section{Methods}

\section{Study site and mosquito collection}

Aedes specimens were sampled during October 2012 in Vietnam in a protected forest of Bù Gia M p National Park located in the province of Binh Phuoc $\left(12^{\circ} 6^{\prime} 42^{\prime \prime} \mathrm{N}\right.$, $107^{\circ} 9^{\prime} 29^{\prime \prime} \mathrm{E}$ according to the World Geodetic System 1984). After several investigations in different habitats of the site, adults of Aedes spp. were only found at the edge of the primary forest inside the protected area (Fig. 7).
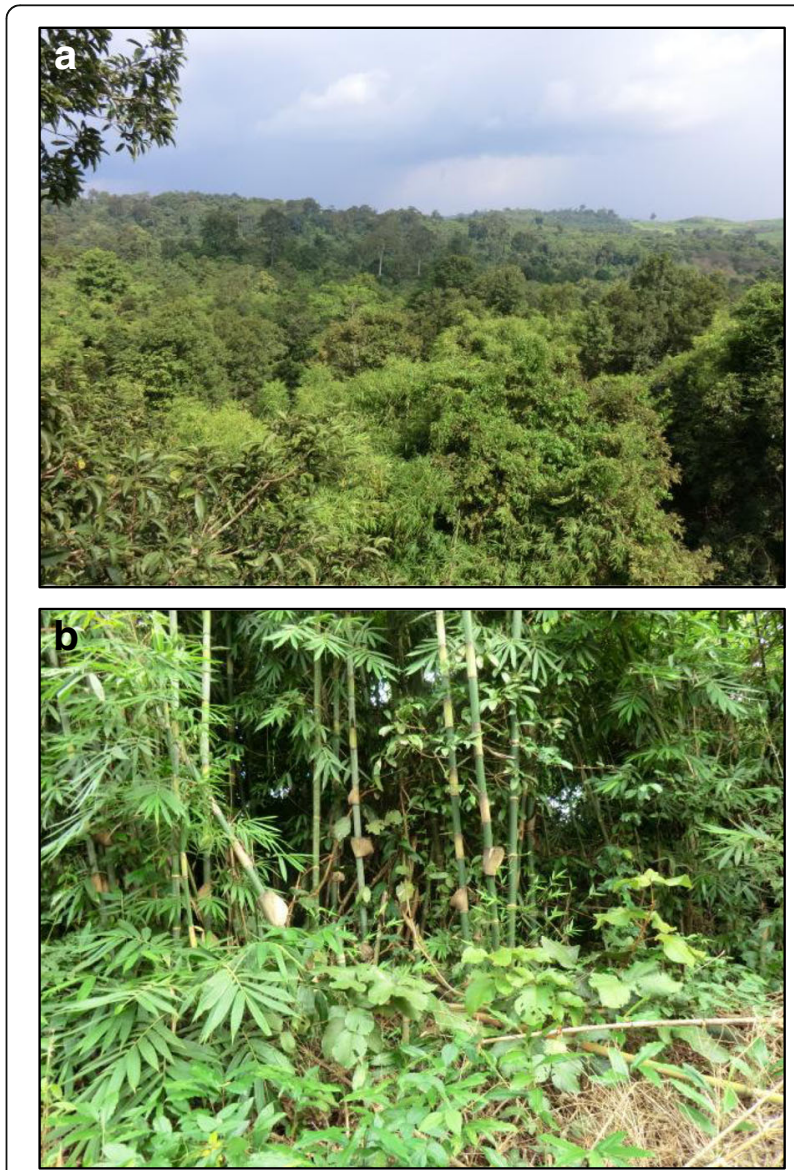

Fig. 7 Bù Gia Mập National Park. a Canopy of the protected forest. b Breeding site where Ae. albopictus mosquitoes were identified and collected 
All collected individuals shared the same breeding sites. Live adult females were caught with nets. Mosquitoes were anesthetized with ether and the morphology of females was immediately observed in the field under a binocular microscope. Each mosquito individual was identified using morphological identification keys for Ae. albopictus $[2-4,9]$ as well as recommendations of the Walter Reed institute (Walter Reed Biosystematic Unit). Only females that could be seen to contain no blood upon microscopic observation of the gut contents were retained for analysis. Mosquitoes were stored in 100\% ethanol at $-80{ }^{\circ} \mathrm{C}$ until used.

\section{Morphological identification}

Photographs of the scutellum and the head were made from 3 individuals of each of the two sub-species according to their genetic structure using nuclear and mitochondrial markers. The mouthparts of specimens were also analysed under a scanning electron microscope (SEM). The preparation of the mosquito's cibarial armature followed an adopted method given by Sirivanakarn [41]. The head of each female was cut off with a sharp razor blade and macerated with $10 \% \mathrm{KOH}$ at $95{ }^{\circ} \mathrm{C}$ until clearance. After clearance, heads were washed in a sodium chloride solution and then dissected with dissecting needles under a binocular microscope in a drop of the sodium chloride solution. Thereafter, the compound eyes were pulled apart to uncover the cibarium with the cibarial armature. For SEM, the samples were subsequently mounted on stubs using a drop of ethanol and then sputter-coated with gold. The samples were analyzed using a Hitachi S-45000 SEM scanning electron microscope. All photographs were compared to Ae. albopictus individuals from sampling sites of Vietnam and France.

\section{Amplification and analysis of mitochondrial markers}

Mosquito DNA extraction was performed following our previous optimized protocol for individual whole mosquitoes [21]. A total of 43 individuals were analyzed (Tables 1 and 2). A 597-bp fragment flanking the mitochondrial cytochrome $c$ oxidase subunit 1 ( $\operatorname{cox} 1)$ gene and a 450-bp fragment flanking the mitochondrial nicotine adenine dinucleotide dehydrogenase subunit 5 (nad5) gene were amplified by polymerase chain reaction (PCR) using $45 \mathrm{ng}$ of DNA matrix as previously described [42]. PCR products were visualised on $1 \%$ agarose gels stained with ethidium bromide. Amplicons were sent for sequencing after purification procedure at a commercial laboratory (Biofidal, Lyon, France). The ITS1-5.8S-ITS2 region was amplified using the universal primers 18SFIN (5'-GTA AGC TTC CTT TGT ACA CAC CGC CCG T-3') and CP16 (5'-GCG GGT ACC ATG CTT AAA TTT AGG GGG TA-3') complementary to highly conserved sequences in the $18 \mathrm{~S}$ and $28 \mathrm{~S}$ rRNA [43]. Reactions were performed with $15 \mathrm{ng}$ DNA, $1.5 \mathrm{mM}$ $\mathrm{MgCl}_{2}, 1 \times$ Reaction buffer, $0.1 \mathrm{mM} \mathrm{dNTP}, 0.24 \mu \mathrm{M}$ of each primer and $1.25 \mathrm{U}$ Taq DNA polymerase (Invitrogen, Cergy Pontoise, France). Amplifications were performed using following cycle conditions: an initial denaturing step at $97{ }^{\circ} \mathrm{C}$ for $4 \mathrm{~min}$; 30 cycles of denaturation at $96{ }^{\circ} \mathrm{C}$ for $30 \mathrm{~s}$, annealing at $48{ }^{\circ} \mathrm{C}$ for $30 \mathrm{~s}$, extension at $72{ }^{\circ} \mathrm{C}$ for $2 \mathrm{~min}$, followed by a final extension at $72{ }^{\circ} \mathrm{C}$ for $4 \mathrm{~min}$. Amplification products were ligated into the TOPO 2.1 vector and transformed into competent One Shot cells using the TOPO TA cloning kit (Invitrogen). DNA inserts were sequenced on both strands. The two strands were aligned and sequencing errors were manually corrected. The sequences were then aligned using MUSCLE [44] algorithm through SEAVIEW software [45] and polymorphic haplotypes were determined using DnaSP v5.10 [46]. The haplotype (Hd) and nucleotidic $(\pi)$ diversities were estimated with DnaSP v5.10. Each haplotype was submitted to GenBank under accession numbers KX495902-KX495951, KX573910-KX573911.

\section{Phylogenetic analysis}

Sequences of the cox 1 and nad5 genes and ITS2 region were used to study the evolutionary relationships between the individuals sampled in the forest of the National Park at Bù Gia Mập. Sequences from different species of the family Culicidae were selected in the GenBank database and used to build the phylogenetic trees. The nad5 phylogenetic tree was built using sequences of Ae. albopictus (gi|374252068), Ae. notoscriptus (gi|508599694, gi|508599680, gi|508599936, gi|508599982, gi|508599786, gi|508599774, gi|508599736, gi|508599844, gi|508599696), Ae. vexans (gi|291193228, gi|854892238, gi|291193230, gi|291193094, gi|291192962), Ae. aegypti (gi|66351510, gi|66351520, gi|66351512, gi|66351506, gi|164370713), Culex pipiens (gi|336462537, gi|336462529, gi|336462535, gi|336462539), Anopheles gambiae (gi|1469 9845, gi|459680999, gi|90656784), An. albitarsis (gi|3093 99294), An. deaneorum (gi|309399336), An. oryzalimnetes (gi|309399308), An. arabiensis (gi|751761052), An. minimus (gi|440655022, gi|440655020), An. punctulatus (gi|3983 14417), An. hinesorum (gi|398314375), An. darling (gi|260 765279), An. funestus (gi|89112084). The cox1 phylogenetic tree was built using sequences of Ae. flavopictus (gi|966 028896, gi|966028894, gi|966028892, gi|474421143, gi $\mid 474$ 421139, gi|474420887, gi|474420611, gi|474421296, gi $\mid 474$ 420608, gi|474420584), Ae. lineatopennis (gi|474420638, gi|474420641, gi|966028902), Ae. cretinus (gi|444435802), Ae. mcintoshi (gi|685228218, gi|685228170, gi|685228140, gi|685228156, gi|685228154), Ae. daiten (gi|474420480), Ae. riversi (gi|474421058, gi|474420596, gi|474420581), Ae. 
cogilli (gi|549534217), Ae. scutellaris (gi|806476819, gi|806 476815), Ae. togoi (gi|844621172), Ae. luniline (gi|6711 84504), Ae. vexans (gi|951294276), Ae. lilii (gi|961557185), Ae. cinereus (gi|953287741, gi|674842465, gi|674842463), Ae. aegypti (gi|806458995, gi|806458969, gi|806458897, gi|347515179), Cx. mimeticus (gi|474420948), Cx. sitiens (gi|474420853), Cx. erythrothorax (gi|861455290), Cx. inflantulus (gi|474420520), Cx. orientalis (gi|966028824), Cx. declarator (gi|861455288), Cx. pipiens (gi|674842155, gi|585421109), An. tesselatus (gi|474420649), An. aquasalis (gi|440494782), An. beklemishevi (gi|374843442, gi|3748 43444, gi|374843430), An. gambiae (gi|292397399, gi|1111 83087). The ITS1-5,8S-ITS2 phylogenetic tree was built using sequences of Ae. simpsoni (gi|16930736, gi|16930732, gi|5566128, gi|1693072), Ae. aegypti (gi|159568), Ae. ochraceus (gi|685228632, gi|685228628), Ae. mcintoshi (gi|685 228596), Cx. quinquefasciatus (gi|306992166), Cx. pipiens (gi|722301, gi|722294), An. clowi (gi|117936018, gi|117 936017), An. farauti (gi|117935967), An. annulipes (gi|117 936015, gi|117936014, gi|117936013). The likelihood of evolutionary model was estimated with jModelTest2 [47] using the Akaike Information Criterion corrected (AICc) and the Bayesian Information Criterion (BIC). Phylogenetic analyses were conducted with MrBayes v.3.2 [48]. Three independent Montecarlo Markov chains (MCMC) were run with 5,000,000 generations. A total of 1,750,000 trees were removed ('burn-in' step) and 3,250,000 trees per replicate were used to estimate the posterior probabilities and the consensus tree. The Potential Scale Reduction Factor confirmed the convergence of the chains.

\section{Detection of Wolbachia in whole individuals}

Wolbachia infection status was evaluated in whole bodies of 43 mosquitoes by PCR detection of wsp gene (Tables 1 and 2). The PCR reaction mixture were performed in reaction volume of $25 \mu \mathrm{l}$ which contained $1 \times$ PCR buffer, $1.5 \mathrm{mM}$ of $\mathrm{MgCl}_{2}, 0.2 \mu \mathrm{M}$ of each primer, $40 \mu \mathrm{M}$ of dNTP, $4 \mathrm{ng} / \mu \mathrm{l}$ of Bovin Serum Albumin (New England Biolabs, Ipswich, USA), 0.02 $\mathrm{U} / \mu \mathrm{l}$ of Taq polymerase (Life Technologies, Saint-Aubin, France). The two generalist wsp primers $81 \mathrm{~F}\left(5^{\prime}\right.$-TGG TCC AAT AAG TGA TGA AGA AAC-3') and 691R (5'AAA AAT TAA ACG CTA CTC CA-3') were used [49]. The amplification protocol was held at $95{ }^{\circ} \mathrm{C}$ for 3 min, followed by 35 amplification cycles at $94{ }^{\circ} \mathrm{C}$ for $45 \mathrm{~s}, 52{ }^{\circ} \mathrm{C}$ for $40 \mathrm{~s}, 72{ }^{\circ} \mathrm{C}$ for $1 \mathrm{~min}$ and a final extension of $72{ }^{\circ} \mathrm{C}$ for $10 \mathrm{~min}$. Amplified fragments of $611 \mathrm{bp}$ were revealed under UV light after migration on $1 \%$ agarose gel electrophoresis. A positive control (DNA from C6/36 cell lines infected with Wolbachia $w \mathrm{AlbB}$ ) and a negative control (DNA from uninfected C6/36 cell lines) were used.

\section{Analysis of midgut bacterial microbiota by b-ARISA}

An additional set of 24 individuals were surface disinfected with $70 \%$ ethanol and rinsed with sterile water as described (Table 1). All dissection steps were performed under a sterile laminar flow hood in a containment environment and midgut DNA extracted as described [22]. Bacterial Automatic Ribosomal Intergenic Spacer Analysis (b-ARISA) was performed following the method of Cardinale et al. [50] with modifications. Reaction mixtures contained 1× of Q5 buffer (New England Biolabs, Ipswich, USA), $1 \times$ of HighGC buffer (New England Biolabs, Ipswich, USA), $0.5 \mu \mathrm{M}$ of each primers (Invitrogen), $200 \mu \mathrm{M}$ of dNTP (Applied Biosystem, Waltham, USA), $120 \mu \mathrm{g} / \mathrm{ml}$ of Bovine Serum Albumin (New England Biolabs, Ipswich, USA), $0.06 \mathrm{mg} / \mathrm{ml}$ of T4 gene 32 and $0.7 \mathrm{U}$ of Q5 DNA polymerase (New England Biolabs, Ipswich, USA) and $30 \mathrm{ng}$ of template DNA in a final volume of $25 \mu \mathrm{l}$. The primers were ITSF (5'-FAMGTC GTA ACA AGG TAG CCG TA-3') and ITSReub (5'-GCC AAG GCA TCC ACC-3') and primer ITSF was $5^{\prime}$ end-labelled with the phosphoramidite dye 6-FAM. Reaction mixtures were held at $94{ }^{\circ} \mathrm{C}$ for $3 \mathrm{~min}$, followed by 35 cycles of amplification at $94{ }^{\circ} \mathrm{C}$ for $45 \mathrm{~s}, 55{ }^{\circ} \mathrm{C}$ for $1 \mathrm{~min}$, and $72{ }^{\circ} \mathrm{C}$ for $1 \mathrm{~min} 20 \mathrm{~s}$ and a final extension of $72{ }^{\circ} \mathrm{C}$ for $1 \mathrm{~min} 20 \mathrm{~s}$. Three replicates per sample were necessary to obtain a sufficient amount of matrix to analyse. Bacterial DNA (Micrococcus sp.) and water were used as positive and negative controls, respectively. The PCR products were purified with QIAquick PCR Purification Kit (Qiagen, Hilden, Germany) and 80 ng of PCR product was loaded with $3 \mu \mathrm{l}$ of Hi-Di-Formamide and $1 \mu \mathrm{l}$ of GS1200 LIZ internal size standard. Sizing tables were obtained with Genemapper 4.0 (Life technologies, Saint-Aubin, France) and imported in $\mathrm{R}$ software ( $\mathrm{R}$ development core team). Only signals within the range of 100-1000 bp were considered for this study. Pick areas were transformed to Relative Fluorescence Intensity (Pick Area $/ \Sigma_{\text {Pick }}$ Area $)$ with the interactive binner and an optimal window size of $5 \mathrm{bp}$ with a shift of $1 \mathrm{bp}$ was selected according to the method previously described [51] (Additional file 2: Table S3). $\alpha$ and $\beta$-diversity analysis were performed with vegan package. DNA extraction was also performed from the remaining carcasses of each individual and ITS15.8S-ITS2 region was amplified as described above. Samples were then assigned to a clade according to the size of amplified fragments after migration of PCR products on a $1 \%$ agarose gel electrophoresis.

\section{MiSeq 16S rDNA gene sequencing}

To determine the composition of midgut associated bacterial microbiota and their diversity, we also used the Illumina MiSeq sequencing technique of the 16S V5-V6 rDNA gene region as previously described [22]. The 
three Ae. albopictus individuals are part of a previous study performed with the same set of samples and their raw data reads are accessible from the European Nucleotide Archive (www.ebi.ac.uk/ena) under the accession number PRJEB6896 [22]. Two individuals corresponding to the cryptic species were included in this analysis (Table 1). Briefly, midgut DNA was extracted as previously described [22]. Libraries were built with one step of PCR conducted with 784 F (5'-AGG ATT AGA TAC CCT GGT A-3') and 1061R (5'-CRR CAC GAG CTG ACG AC-3') modified with an 8-bp multiplex barcode and Illumina adapters. The PCR mixture contained 1.75 U of Expand High Fidelity Enzyme Mix (Roche, Basel, Switzerland), 1× of Expand High Fidelity Buffer (Roche, Switzerland), $0.06 \mathrm{mg} \cdot \mathrm{ml}^{-1}$ of $\mathrm{T} 4$ gene 32 protein (New England Biolabs, France), $40 \mu \mathrm{M}$ of dNTP mix, $200 \mathrm{nM}$ of each primers (Life Technologies, France). Amplifications were conducted with $5 \mathrm{~min}$ at $95{ }^{\circ} \mathrm{C}$ followed by 40 cycles at $95{ }^{\circ} \mathrm{C}$ for $40 \mathrm{~s}, 54.2{ }^{\circ} \mathrm{C}$ for $1 \mathrm{~min}, 72{ }^{\circ} \mathrm{C}$ for $30 \mathrm{~s}$ and a final extension at $72{ }^{\circ} \mathrm{C}$ for $7 \mathrm{~min}$. Three PCR per sample were pooled together and equimolar product were mixed for all the samples libraries after purification with the Agencourt AMPure XP PCR Purification kit (Beckman Coulter, Villepinte, France). Sequencing was performed on the Illumina MiSeq platform $(2 \times 250 \mathrm{bp}$ paired-end reads) by ProfileXpert - ViroScan 3D (Lyon, France). The complementary reads were aligned with PandaSeq [52]. Aligned sequences were then processed with the MOTHUR pipeline and were kept only if: (i) their size was comprised between 200 and 350 bp; (ii) they did not contain any ambiguous sequence; (iii) they were not chimeric according to Perseus chimera detection; (iv) they aligned on the SILVA database (released 115). OTUs were created by clustering sequences at a level of $97 \%$ similarity according to the median neighbour method [53]. The sequences were classified with a naïve Bayesian classifier at an $80 \%$ bootstrap [54]. A total of 51843, 81088, 32279, 69453 and 34077 sequences were obtained for the samples BGM1, BGM3, BGM4, BGM5 and BGM6, respectively. A negative control (blank extraction and PCR) was used as a reference to remove the contaminants as previously described [22]. The sequence proportions were obtained after subsampling of 8002 sequences per sample. Because of the small samples size, no comparative analysis of the $\alpha$ and $\beta$ diversity was performed. Only the proportions of OTUs assignment were presented. The raw reads related to the two individuals from the clade $\mathrm{C}$ have been deposited in the European Nucleotide Archive (www.ebi.ac.uk/ena) under the accession number PRJEB14610. DNA extraction, cox1 and nad5 barcoding were performed on the carcasses of those individuals according to the protocol previously described.

\section{Additional files}

Additional file 1: Figure S1. Correlation of pairwise nucleotidic distances for cox1, nad5 and ITS1-5.8S-ITS2 markers. Figure S2. Molecular features reveal differences between Ae. albopictus and a cryptic Aedes species living in sympatry. Table S1. Analysis of haplotype and nucleotide diversity within the two Aedes clades. Table S2. Proportions of operational taxonomic units (OTU) of bacteria identified in midgut samples by 165 rDNA Miseq sequencing. (PDF $1193 \mathrm{~kb}$ )

Additional file 2: Table S3. b-ARISA dataset. (XLSX $25 \mathrm{~kb}$ )

\section{Abbreviations}

$16 S$ rDNA: 165 ribosomal DNA; CHICKV: Chickungunya virus; cox1: Cytochrome c oxidase subunit 1; DENV: Dengue virus; Hd: Haplotype diversity; ITS: Internal transcribed spacer; K2P: Kimura 2-parameter; nad5: Nicotinamide adenine dinucleotide dehydrogenase subunit 5

\section{Acknowledgments}

This paper is dedicated to the memory of Florence-Hélène Tran who tragically left us in August 2016. The authors thank the Vietnamese authorities, director and staff of Bù Gia M p National Park for giving them the permission to sample Culicidae. We thank Dr Clement Goubert from the University of Lyon for his help to identify the cryptic species. We also sincerely thank Dr Abilash Nair from the University of Helsinki and Dr Pablo Tortosa from the Reunion University for their valuable advice.

\section{Funding}

Funding for this project was provided by grants from EC2CO CNRS and CMIRA Région Rhône-Alpes. This research was also partially funded by ERA-NET BiodivERsA with the national funders ANR-13-EBID-0007-01, FWF I-1437, and DFG KL 2087/6-1 as part of the 2012-2013 BiodivERsA call for research proposals, and was carried out within the framework of GDRI "Biodiversity and Infectious Diseases in Southeast Asia". We also gratefully acknowledge the contribution of the DTAMB platform of the FR41 Bio-Environment and Health (University Lyon 1) and the Academy of Finland (Decision numbers 273098 and 265641).

Availability of data and materials

All data generated or analysed during this study are included in this published article and its Additional files.

\section{Authors' contributions}

GM, PM and CVM conceived the project and sampling design. GM and CVM wrote the first draft of the manuscript. GM, VTV, KLHK, THTT, HTN, PP, PM and CVM contributed to collection of specimens. GM, CVM, F-HT and VTV performed all molecular work and analyzed and interpreted the data. CM, SK and LK prepared the specimens for morphological identification and scanning electron microscopy observation. All authors read and approved the final manuscript.

\section{Consent for publication}

Not applicable.

\section{Competing interests}

The authors declare that they have no competing interests.

Ethics approval and consent to participate

Not applicable.

\section{Publisher's Note}

Springer Nature remains neutral with regard to jurisdictional claims in published maps and institutional affiliations. 


\section{Author details}

${ }^{1}$ Université de Lyon, Lyon, France. ${ }^{2}$ Université Lyon 1, Villeurbanne, France. ${ }^{3}$ CNRS, UMR 5557, Ecologie Microbienne, Villeurbanne, France. ${ }^{4}$ INRA, UMR1418, Villeurbanne, France. ${ }^{5}$ Metapopulation Research Center, Department of Biosciences, University of Helsinki, Helsinki, Finland. ${ }^{6}$ Institute for Ecology, Evolution and Diversity, Goethe-University, Frankfurt am Main, Germany. 'Senckenberg Biodiversity and Climate Research Centre, Senckenberg Gesellschaft für Naturforschung, Frankfurt am Main, Germany. ${ }^{8}$ Department of Medical Entomology and Zoonotics, Pasteur Institute in Ho Chi Minh City, Ho Chi Minh City, Vietnam. ${ }^{9}$ Université de La Réunion, CNRS 9192, INSERM U1187, IRD 249, Unité Mixte Processus Infectieux en Milieu Insulaire Tropical (PIMIT), Plateforme Technologique CYROI, Sainte-Clotilde, La Réunion, France.

Received: 1 February 2017 Accepted: 17 May 2017 Published online: 02 June 2017

\section{References}

1. Mosquito taxonomic inventoy. 2016. http://mosquito-taxonomic-inventory. info. Accessed 1 Jul 2016.

2. Barraud PJ. A revision of the culicine mosquitoes of India, Part XXIII. The genus Aedes (sens. lat.) and the classification of the subgenus. Descriptions of the Indian species of Aedes (Aedimorphus), Aedes (Ochlerotatus), and Aedes (Banksinella), with notes on Aedes (Stegomyia) uariegatus. Ind J Med Res. 1928;15:653-69.

3. Huang Y-M. Contributions to the mosquito fauna of Southeast Asia. XIV. The subgenus Stegomyia of Aedes in Southeast Asia I - The scutellaris group of species. Contrib Am Ent Inst. 1972;9:1-109.

4. Tanaka K, Mizusawa K, Saugstad ES. A revision of the adult and larval mosquitoes of Japan (including the Ryukyu Archipelago and the Ogasawara Islands) and Korea (Diptera: Culicidae). San Francisco: Contributions of the American Entomological Institute (Ann Arbor); 1979. p. 1-987.

5. Rueda LM. Pictorial keys for the identification of mosquitoes (Diptera: Culicidae) associated with dengue virus transmission. Walter Reed army inst. of research. Washington: Department of Entomology; 2004.

6. Hawley WA. The biology of Aedes albopictus. J Am Mosq Control Assoc Suppl. 1988;1:1-39.

7. Huang Y-M. Medical Entomology Studies - XI. The subgenus Stegomyia of Aedes in the Oriental region with keys to the species (Diptera: Culicidae). Medical Entomology Project Report. Washington: Smithsonian Institution; 1979.

8. McLain DK, Rai KS, Fraser MJ. Intraspecific and interspecific variation in the sequence and abundance of highly repeated DNA among mosquitoes of the Aedes albopictus subgroup. Heredity. 1987;58(Pt 3):373-81.

9. Patsoula E, Samanidou-Voyadjoglou A, Spanakos G, Kremastinou J, Nasioulas G, Vakalis NC. Molecular and morphological characterization of Aedes albopictus in northwestern Greece and differentiation from Aedes cretinus and Aedes aegypti. J Med Entomol. 2006:43:40-54.

10. Rani A, Sharma A, Rajagopal R, Adak T, Bhatnagar RK. Bacterial diversity analysis of larvae and adult midgut microflora using culture-dependent and culture-independent methods in lab-reared and field-collected Anopheles stephensi - an Asian malarial vector. BMC Microbiol. 2009;9:96.

11. Gimonneau G, Tchioffo MT, Abate L, Boissière A, Awono-Ambéné PH, Nsango SE, et al. Composition of Anopheles coluzzii and Anopheles gambiae microbiota from larval to adult stages. Infect Genet Evol. 2014;28:715-24.

12. Muturi EJ, Kim C-H, Bara J, Bach EM, Siddappaji MH. Culex pipiens and Culex restuans mosquitoes harbor distinct microbiota dominated by few bacterial taxa. Parasit Vectors. 2016;9:18.

13. Duguma D, Rugman-Jones $P$, Kaufman MG, Hall MW, Neufeld JD, Stouthamer R, et al. Bacterial communities associated with Culex mosquito larvae and two emergent aquatic plants of bioremediation importance. PLoS One. 2013;8:e72522.

14. Minard G, Mavingui $P$, Moro CV. Diversity and function of bacterial microbiota in the mosquito holobiont. Parasit Vectors. 2013;6:146.

15. Engel P, Moran NA. The gut microbiota of insects - diversity in structure and function. FEMS Microbiol Rev. 2013;37:699-735.

16. Shapira M. Gut microbiotas and host evolution: scaling up symbiosis. Trends Ecol Evol. 2016:31:539-49.

17. Kittayapong P, Baisley KJ, Sharpe RG, Baimai V, O'Neill SL. Maternal transmission efficiency of Wolbachia superinfections in Aedes albopictus populations in Thailand. Am J Trop Med Hyg. 2002;66:103-7.
18. Zouache K, Raharimalala FN, Raquin V, Tran-Van V, Raveloson LHR, Ravelonandro P, et al. Bacterial diversity of field-caught mosquitoes, Aedes albopictus and Aedes aegypti, from different geographic regions of Madagascar. FEMS Microbiol Ecol. 2011;75:377-89.

19. de Albuquerque AL, Magalhães T, Ayres CFJ. High prevalence and lack of diversity of Wolbachia pipientis in Aedes albopictus populations from Northeast Brazil. Mem Inst Oswaldo Cruz. 2011;106:773-6.

20. Bourtzis K, Dobson SL, Xi Z, Rasgon JL, Calvitti M, Moreira LA, et al. Harnessing mosquito-Wolbachia symbiosis for vector and disease control. Acta Trop. 2014. doi:10.1016/j.actatropica.2013.11.004.

21. Minard G, Tran F-H, Dubost A, Tran-Van V, Mavingui P, Valiente MC. Pyrosequencing $16 \mathrm{~S}$ rRNA genes of bacteria associated with wild tiger mosquito Aedes albopictus: a pilot study. Front Cell Infect Microbiol. 2014;4:59.

22. Minard G, Tran FH, Van VT, Goubert C, Bellet C, Lambert G, et al. French invasive Asian tiger mosquito populations harbor reduced bacterial microbiota and genetic diversity compared to Vietnamese autochthonous relatives. Front Microbiol. 2015:6:970.

23. Dobson SL, Marsland EJ, Rattanadechakul W. Mutualistic Wolbachia infection in Aedes albopictus: accelerating cytoplasmic drive. Genetics. 2002;160:1087-94.

24. Bordenstein SR, O'Hara FP, Werren JH. Wolbachia-induced incompatibility precedes other hybrid incompatibilities in Nasonia. Nature. 2001:409:707-10.

25. Rasgon JL, Gamston CE, Ren X. Survival of Wolbachia pipientis in cell-free medium. Appl Env Microbiol. 2006;72:6934-7.

26. Brucker RM, Bordenstein SR. The hologenomic basis of speciation: gut bacteria cause hybrid lethality in the genus Nasonia. Science. 2013;341:667-9.

27. Kenney JL, Brault AC. Chapter two. The role of environmental, virological and vector interactions in dictating biological transmission of arthropodborne viruses by mosquitoes. Adv. Virus Res. 2014;89:39-83.

28. Forrester NL, Coffey LL, Weaver SC. Arboviral bottlenecks and challenges to maintaining diversity and fitness during mosquito transmission. Viruses. 2014:6:3991-4004

29. Jupatanakul N, Sim S, Dimopoulos G. The insect microbiome modulates vector competence for arboviruses. Viruses. 2014;6:4294-313.

30. Takano KT, Nguyen NTH, Nguyen BTH, Sunahara T, Yasunami M, Nguyen $M D$, et al. Partial mitochondrial DNA sequences suggest the existence of a cryptic species within the leucosphyrus group of the genus Anopheles (Diptera: Culicidae), forest malaria vectors, in northern Vietnam. Parasit Vectors. 2010;3:41.

31. Cuong HQ, Vu NT, Cazelles B, Boni MF, Thai KTD, Rabaa MA, et al. Spatiotemporal dynamics of dengue epidemics, southern Vietnam. Emerg Infect Dis. 2013;19:945-53.

32. Rasgon JL, Cornel AJ, Scott TW. Evolutionary history of a mosquito endosymbiont revealed through mitochondrial hitchhiking. Proc Roy Soc B Biol Sci. 2006:273:1603-11.

33. Atyame CM, Delsuc F, Pasteur N, Weill M, Duron O. Diversification of Wolbachia endosymbiont in the Culex pipiens mosquito. Mol Biol Evol. 2011;28:2761-72.

34. Dumas E, Atyame CM, Malcolm CA, Le Goff G, Unal S, Makoundou P, et al Molecular data reveal a cryptic species within the Culex pipiens mosquito complex. Insect Mol Biol. 2016;25:800-9.

35. Hurst GD, Jiggins FM. Problems with mitochondrial DNA as a marker in population, phylogeographic and phylogenetic studies: the effects of inherited symbionts. Proc Roy Soc B Biol Sci. 2005;272:1525-34.

36. Sun X-J, Xiao J-H, Cook JM, Feng G, Huang D-W. Comparisons of host mitochondrial, nuclear and endosymbiont bacterial genes reveal cryptic fig wasp species and the effects of Wolbachia on host mtDNA evolution and diversity. BMC Evol Biol. 2011;11:86.

37. Ritter S, Michalski SG, Settele J, Wiemers M, Fric ZF, Sielezniew M, et al. Wolbachia infections mimic cryptic speciation in two parasitic butterfly species, Phengaris teleius and P. nausithous (Lepidoptera: Lycaenidae). PLoS One. 2013:8:e78107.

38. Vaz-Moreira I, Nunes OC, Manaia CM. Diversity and antibiotic resistance patterns of Sphingomonadaceae isolates from drinking water. Appl Environ Microbiol. 2011;77:5697-706.

39. Ashton AQ. Advances in Sphingomonadaceae research and application. Atlanta: ScholarlyEditions; 2012.

40. Derycke S, De Meester N, Rigaux A, Creer S, Bik H, Thomas WK, et al. Coexisting cryptic species of the Litoditis marina complex (Nematoda) show differential resource use and have distinct microbiomes with high intraspecific variability. Mol Ecol. 2016;25:2093-110. 
41. Sirivanakarn S. The Female cibarial armature of new world Culex, subgenus Melanoconion and related subgenera with notes on this character in subgenera Culex, Lutzia and Neoculex and genera Galindomyia and Deinocerites. Medical entomology project report. Washington: Smithsonian Institution; 1978.

42. Raharimalala FN, Ravaomanarivo LH, Ravelonandro P, Rafarasoa LS, Zouache K, Tran-Van V, et al. Biogeography of the two major arbovirus mosquito vectors, Aedes aegypti and Aedes albopictus (Diptera, Culicidae), in Madagascar. Parasit Vectors. 2012;5:56

43. Higa Y, Toma T, Tsuda Y, Miyagi I. A multiplex PCR-based molecular identification of five morphologically related, medically important subgenus Stegomyia mosquitoes from the genus Aedes (Diptera: Culicidae) found in the Ryukyu Archipelago. Japan Jpn J Infect Dis. 2010;63:312-6.

44. Edgar RC. MUSCLE: multiple sequence alignment with high accuracy and high throughput. Nucleic Acids Res. 2004;32:1792-7.

45. Gouy M, Guindon S, Gascuel O. SeaView version 4: a multiplatform graphical user interface for sequence alignment and phylogenetic tree building. Mol Biol Evol. 2010;27:221-4.

46. Librado P, Rozas J. DnaSP v5: a software for comprehensive analysis of DNA polymorphism data. Bioinforma. 2009;25:1451-2.

47. Darriba D, Taboada GL, Doallo R, Posada D. jModelTest 2: more models, new heuristics and high-performance computing. Nat Methods. 2012;9:772.

48. Ronquist F, Teslenko M, van der Mark P, Ayres DL, Darling A, Höhna S, et al. MrBayes 3.2: efficient bayesian phylogenetic inference and model choice across a large model space. Syst Biol. 2012;61:539-42.

49. Zhou W, Rousset F, O'Neil S. Phylogeny and PCR-based classification of Wolbachia strains using wsp gene sequences. Proc Roy Soc B Biol Sci. 1998;265:509-15.

50. Cardinale M, Brusetti L, Quatrini P, Borin S, Puglia AM, Rizzi A, et al. Comparison of Different primer sets for use in automated ribosoma intergenic spacer analysis of complex bacterial communities. Appl Environ Microbiol. 2004;70:6147-56.

51. Ramette A. Quantitative community fingerprinting methods for estimating the abundance of operational taxonomic units in natural microbial communities. Appl Environ Microbiol. 2009:75:2495-505.

52. Masella AP, Bartram AK, Truszkowski JM, Brown DG, Neufeld JD. PANDAseq: paired-end assembler for illumina sequences. BMC Bioinformatics. 2012;13:31.

53. Schloss PD, Westcott SL, Ryabin T, Hall JR, Hartmann M, Hollister EB, et al. Introducing mothur: open-source, platform-independent, communitysupported software for describing and comparing microbial communities. Appl Environ Microbiol. 2009;75:7537-41.

54. Wang Q, Garrity GM, Tiedje JM, Cole JR. Naïve Bayesian classifier for rapid assignment of rRNA sequences into the new bacterial taxonomy. Appl Environ Microbiol. 2007;73:5261-7.

\section{Submit your next manuscript to BioMed Central and we will help you at every step:}

- We accept pre-submission inquiries

- Our selector tool helps you to find the most relevant journal

- We provide round the clock customer support

- Convenient online submission

- Thorough peer review

- Inclusion in PubMed and all major indexing services

- Maximum visibility for your research

Submit your manuscript at www.biomedcentral.com/submit 